\title{
Coping with the coronavirus (COVID-19) pandemic
}

\author{
Rüstem Aşkın $\odot$, Ezgi Tan Dinselø, Alper Baş® \\ Department of Psychology, Istanbul Commerce University, Faculty of Humanities and Social Sciences, İstanbul, Turkey
}

\begin{abstract}
The global virus outbreak called COVID-19 has been one of the important breaking points in world history. COVID-19 is not only a physical disease affecting the whole world, but also an important issue in terms of community mental health, the effects of which will be understood in the long term. It is essential to explain the long-term and ongoing mental health effects of COVID-19 to take preventive steps. The aim of the present article was to review the impact of similar outbreaks in the past and to understand the potential impact on mental health through data from ongoing studies. Additionally, we summarized possible risk factors that may arise in infected people, healthcare professionals and the general population during COVID-19 outbreak.

Keywords: Covid-19, mental health, psychiatry, depression, anxiety, healthcare professionals
\end{abstract}

C OVID-19 outbreak was a sudden pandemic for almost any country, society or family. With the advances in medicine, the use of antibiotics, vaccines and sanitation, the ever-decreasing deadly and infectious disease threat reappeared with the COVID-19 outbreak [1]. In the midst of an unusual infectious outbreak, fear and negative attitudes among people are understandable since being infected is not affected by any gender and socio-demographic situation and it is a potential risk for everyone.

Throughout history, outbreaks have had lasting and massive effects, from handover of dynasties to colonialism and even climate change. Many epidemics such as bubonic plague, Justinian plague, smallpox, Spanish flu, Asian flu, scarlet fever and rubella have left important trace in history and have significantly affected the course of events. In recent years, epidemic diseases such as SARS, MERS, bird flu, Swine flu, Ebola and new type coronavirus (Covid-19) spread rapidly in a short time. Covid-19, which was first seen in December 2019, infected three million people just in four months, causing the death of hundreds of thousands of people.

The earlier reactions to the attack of a virus, the source of which was still not fully understood, were denial, shock and surprise. Denial was initially a defense reaction that all societies had to deal with, reflecting not only ignorance but also the difficulty in accepting such kind of threat since every person on earth was under risk. Especially in the earlier stages of the pandemic, burying of the bodies quickly and randomly, and cremating of them collectively and brutally in the middle of the streets in some countries, were watched with horror by billions of people. Media portrayal has assisted to frighten both "those who were not afraid", and who were already anxious.

Kelly [2] had indicated that some preventive actions to control the virus may have negative effects on mental health of individuals. Due to lack of information, quarantine can be associated with frustration, fear of infection, boredom and anxiety. All schedules, projects, programs, short-medium-long-term plans 
were postponed to an uncertain date during the process we have been living. Stigmatization and financial problems also have increased the risk of potential mental health problems. Covid-19 caused mental health problems in two ways. First, the mental problems caused by the virus itself, and the second is the anxiety, panic and psychological problems associated with the pandemic. Covid-19 should be considered as not only a health crisis but also a mental health emergency. Initially, much less attention has been paid to the mental health consequences of the pandemic than other medical outcomes posed by the viral infection. However, when the pandemic ends; we will resume our normal life, but its psychological effects will probably last for months or even years.

Studies investigating the psychiatric effects of the outbreak on people are few and still ongoing. Therefore, it would be beneficial to examine the effects of past outbreaks or traumatic events on mental health in order to predict potential consequences of COVID-19 pandemic. This paper aims to review current and previous studies to explain the effects of COVID-19 on mental health.

Mental health impacts of COVID-19 on the general population and the infected patients

In China, a web-based cross-sectional survey revealed that prevalence of generalized anxiety disorder (GAD), depressive symptoms and poor sleep quality were $35.1 \%, 20.1 \%$ and $18.2 \%$ respectively during COVID-19 outbreak [3]. GAD and depressive symptoms were more common among participants younger than 35 years. According to American Psychiatric Association's national survey [4], about half of Americans (48\%) were concerned about being infected by COVID-19. 40\% of Americans were concerned about serious illness or death from COVID-19, while $62 \%$ of Americans were concerned about the possibility of their family members or loved ones getting COVID-19 [5]. These studies evaluate the psychological well-being and anxiety/depression levels that have been experienced over past 2 weeks. Unlike other studies, in Denmark results were compared with the previous surveys that had been conducted in 2016. This study showed that the psychological well-being of the general Danish population was negatively affected by the COVID-19 pandemic. When these studies are considered, it is understood that the epidemic might exert negative effects on general well-being of individuals, increase the risk of depression and anxiety.

In China, to examine the potential mental health problems related to quarantine, researchers obtained data from online survey and compared the quarantined and not quarantined groups [6]. High prevalence of mental health problems were found in both groups, however there was no significant difference between participants with or without quarantine. According to that study, mental health problems during this period were due to the effects of COVID-19 on daily life, not due to quarantine.

Zhang et al. [7] compared three different groups to evaluate the psychological distress across populations in Zhongshan, Guangdong China. COVID-19 infected patients who were recovered from illness, quarantined individuals and the general public were compared. Prevalence of depression comorbid with anxiety symptoms in the quarantined group were lower $(8 \%)$ than that of the infected $(21.1 \%)$ and the general public (22.4\%). High prevalence of depression (29.2\%) was found among patients who experienced COVID-19 infection. The prevalence of anxiety was not different among three groups. COVID-19 infected patients $(19.3 \%)$ and general public $(14.3 \%)$ had severe depression symptoms, infected patients and general public were more likely to have depressive mood, anxiety-like behaviors and somatic symptoms.

To evaluate the long-term effects of COVID-19, and whether the outbreak had changed the symptoms of generalized anxiety, post-traumatic stress disorder (PTSD) and depression over time, it had been investigated with the data obtained during the initial outbreak and four weeks later during the peak of the epidemic in China. In the first assessment, moderate to severe levels of anxiety, stress and depression were found in groups. Levels of stress, anxiety, depression, and PTSD symptoms of participants did not differ significantly over time [8].

\section{Mental health of COVID-19 patients and health care workers}

There are studies supporting the view that recent epidemics had severe effects on mental health of the infected patients. It is revealed that mental health problems have occurred among healthcare workers and patients who survived SARS epidemic [9] and 
MERS epidemic [3]. When the long-term effects of the SARS epidemic had been analyzed, it was observed that the prevalence of depressive disorders and PTSD has increased after outbreak $[3,10]$.

Infected people may have feelings of guilt, anxiety and hopelessness combined with physical problems of infection and long periods of hospitalization[2]. Complex emotional processes are likely to occur due to the feelings of guilt, despair, loss and inability to visit patients or limited funerals. In this emotionally charged environment, psychological support is vital both at the time of death, illness and in the future. Mental health workers should be able to reach to infected and quarantined patients to get appropriate mental health services during COVID 19 outbreak.

In addition to infected patients, the mental health of health workers combatting with the virus is also at significant risk. Especially, health care personnel working in the intensive care units and the emergency departments are at risk for psychological symptoms. During SARS epidemic in Singapore, psychiatric symptoms were seen $27 \%$ of health care workers [10], and PTSD symptoms arised among health care workers during MERS outbreak in Korea. Correspondingly, during Ebola outbreak in Congo, health care workers reported high levels of anxiety and fear of being stigmatized [2].

During COVID-19 outbreak, Lai et al. [13] aimed to explore the healthcare workers' psychiatric symptoms. It has been found that the frequency of depression, anxiety, insomnia and distress symptoms were significantly higher among health-care workers who treated COVID-19 patients. Especially health care professionals were exposed patients with COVID-19, experienced higher rates of depression and anxiety compared to those without exposure [14]. Furthermore, the study revealed significantly higher levels of anxiety and depression experienced by pediatric health personnel during the COVID-19 outbreak in China Guiyang.

A similar study had been conducted in China, to examine the work stress of nurses who were working against COVID-19 [15]. The study revealed that stress load of nurses was significantly high and they were experiencing considerable stress during that period of COVID-19 [15]. Li et al. [16] compared general public, medical workers, and medical workers that directly work with COVID-19 patients according to their vicarious traumatization level. Study revealed that vicarious traumatization levels of general public and non-front-line nurses were higher than the nurses that directly deal with infected patients. This study reveals that, not only health care workers but also general population are at risk for traumatizing experiences even they are living in isolation.

\section{Mental health risks of COVID-19 on pre-existing} mental health problems and vulnerable groups

While the mental problems of healthcare workers and infected patients are primarily considered, other groups should also be explored to identify vulnerable populations and risk groups. By this way, it may be possible to take protective measures and track longterm effects. When outbreaks occur, people with mental health disorders are often more vulnerable to infections for several reasons [17]. Cognitive impairment, less risk awareness and less personal protection efforts of people with mental disorders may increase the risk of infectious diseases in everyday life and in psychiatric clinics. Another potential risk factor is that infected people with mental disorders may have low awareness to seek treatment on time or they may have difficulty in getting services due to their mental condition. For some patients with their own idiosyncratic concerns, they may have trouble grasping the reality when a true threat really exists. Compared with the general population, people with mental health disorders may be more affected by emotional conditions caused by COVID-19 outbreak, and their condition may worsen. Therefore, the disease caused by COVID-19 is also a risk factor for psychiatric patients, as the life expectancy and general health outcomes of people with mental illness are lower than general population. On the other hand, many people with mental disorders regularly receive outpatient treatment. However, with the countries' travel restrictions and quarantine procedures, regular doctor checks may become difficult and disrupt treatment processes.

During this period, for some of the groups who resume their consultations through online therapy, social isolation and online therapy contain risk factors in itself. Telepsychiatry can be challenging in paranoid patients, individuals with suicide risk, substance abuse, individuals with a tendency to violent behaviors, and psychotic patients receiving regular medication [18]. 
It is proposed that individuals with suicide risk require special attention during the pandemic period [19]. There are some studies showing that suicide rates increased in the U.S. during the 1918-19 flu outbreak and among older people in Hong Kong during severe acute respiratory syndrome (SARS) outbreak in 2003 [20]. Suicide risk increases when social isolation and loneliness occurs, so during this outbreak, current situation increases suicide risk especially for individuals who are grieving due to loss of a loved one $[19,21]$.

It may be important to understand the COVID-19 process on children with different developmental characteristics. One of such groups that might be in risk during COVID-19 outbreak is children with ADHD. In most countries, schools were closed and students were expected to attend online education. It is anticipated that children with ADHD may face visible difficulties during this period due to the loss of the daily routine, interpersonal and social interaction. According to the results of the first study focusing on children with ADHD during the COVID-19 outbreak, the behaviors of children with ADHD have significantly deteriorated during outbreak compared to their normal state [7]. Additionally, negative correlations were found between the ADHD symptoms and increased online education and working time. According to this cross-sectional research, ADHD symptoms decreased with longer working time.

\section{CONCLUSION}

Ongoing studies have been trying to explore the potential effects of COVID-19 on population mental health. COVID-19 may have an aggravating effect on mental health of populations during and after the pandemic. Therefore, risk factors should be determined for comprehensive interventions in the future, such as poor mental health before the outbreak, being infected or having infected family members, separation from family and social support, low economic conditions. Thus far, under strict infection treatment procedures, unless mandatory situations for psychiatrists, psychologists and social workers, it was difficult to enter isolation services for patients. Therefore, front-line health workers were the main personnel, who also provided psychological support, in addition to their workload [22]. However, the importance and position of psychological support will be more obvious in the near future. Overall, these extraordinary times are thought to make many people vulnerable to mental health problems and risky behaviors and these mental health problems are predicted to be more apparent after the pandemic.

\section{Conflict of interest}

The author disclosed no conflict of interest during the preparation or publication of this manuscript.

\section{Financing}

The authors disclosed that they did not receive any grant during conduction or writing of this study.

\section{REFERENCES}

1. Shalev D, Shapiro PA. Epidemic psychiatry: the opportunities and challenges of COVID-19. Gen Hosp Psychiatry 2020;64:6871.

2. Kelly BD. Covid-19 (Coronavirus): challenges for psychiatry. Br J Psychiatry 2020 Apr 15;1-2. doi: 10.1192/bjp.2020.86

3. Huang Y, Zhao N. Generalized anxiety disorder, depressive symptoms and sleep quality during COVID-19 outbreak in China: a web-based cross-sectional survey. Psychiatry Res 2020;288:112954.

4. American Psychiatric Association. New Poll:COVID-19 ImpactingMentalWell-Being. Available at: www.psychiatry.org/newsroom/news-releases/new-poll-covid19-impacting-mental-well-being-americans-feeling-anxious-espe cially-for-loved-ones-older-adults-are-less-anxious. Accessed March 25, 2020.

5. Sønderskov KM, Dinesen PT, Santini ZI, Østergaard SD. The depressive state of Denmark during the COVID-19 pandemic. Acta Neuropsychiatr 2020 Apr 22;1-3. doi: 10.1017/neu.2020.15. 6. Zhu S, Wu Y, Zhu CY, Hong WC, Yu ZX, Chen ZK, et al. The immediate mental health impacts of the COVID-19 pandemic among people with or without quarantine managements. Brain Behav Immun 2020 Apr 18;S0889-1591(20)30601-2. doi: 10.1016/j.bbi.2020.04.045.

7. Zhang J, Lu H, Zeng H, Zhang S, Du Q, Jiang T, et al. The differential psychological distress of populations affected by the COVID-19 pandemic. Brain Behav Immun 2020 Apr 15;S08891591(20)30535-3. doi: 10.1016/j.bbi.2020.04.031.

8. Wang C, Pan R, Wan X, Tan Y, Xu L, McIntyre RS, et al. Longitudinal study on the mental health of general population during the COVID-19 epidemic in China. Brain Behav Immun $020 \mathrm{Apr}$ 13;S0889-1591(20)30511-0. doi: 10.1016/j.bbi.2020.04.028.

9. Lee AM, Wong JG, McAlonan, GM, Cheung V, Cheung C, Sham PC, et al. Stress and psychological distress among SARS survivors 1 year after the outbreak. Can J Psychiatry 2007;52:233-40.

10. Mak IW, Chu MC, Pan PC, Yiu MG, Chan VL. Long-term 
psychiatric morbidities among SARS survivors. Gen Hosp Psychiatry 2009;31:318-26.

11. Lee SM, Kang WS, Cho AR, Kim T, Park JK. Psychological impact of the 2015 MERS outbreak on hospital workers and quarantined hemodialysis patients. Compr Psychiatry 2018;87:123-7.

12. Park JS, Lee EH, Park NR, Choi YH. Mental health of nurses working at a government-designated hospital during a MERSCoV outbreak: a cross-sectional study. Arch Psychiatr Nurs 2008;32:2-6.

13. Lai J, Ma S, Wang Y, Cai Z, Hu J, Wei N, et al. Factors associated with mental health outcomes among health care workers exposed to coronavirus disease 2019. JAMA Netw Open 2020;3:e203976.

14. Chen Y, Zhou H, Zhou Y, Zhou F. Prevalence of self-reported depression and anxiety among pediatric medical staff members during the COVID-19 outbreak in Guiyang, China. Psychiatry Res 2020;288:113005.

15. Mo Y, Deng L, Zhang L, Lang Q, Liao C, Wang N, et al. Work stress among Chinese nurses to support Wuhan for fighting against the COVID-19 epidemic. J Nurs Manag 2020 Apr 7. doi: 10.1111/jonm.13014.
16. Li Z, Ge J, Yang M, Feng J, Qiao M, Jiang R, et al. Vicarious traumatization in the general public, members, and non-members of medical teams aiding in COVID-19 control. Brain Behav Immun 2020 Mar 10;S0889-1591(20)30309-3. doi: 10.1016/j.bbi.2020.03.007.

17. Yao H, Chen JH, Xu YF. Patients with mental health disorders in the COVID-19 epidemic. Lancet Psychiatry 2020;7:e21. 18. Goldberg, JF. Psychiatry's niche role in the COVID-19 pandemic. J Clin Psychiatry 2020;81:20com13363.

19. Gunnel D, Appleby L, Arensman E, HawtonK, John A, Kapur $\mathrm{N}$, et al. Suicide risk and prevention during the COVID-19 pandemic. Lancet Psychiatry 2020;7:468-71.

20. Wasserman IM. The impact of epidemic, war, prohibition and media on suicide: United States, 1910-1920. Suicide Life Threat Behav1992;22:240-54.

21. O'Connor RC, Kirtley OJ. The integrated motivational-volitional model of suicidal behaviour. Philos Trans R Soc Lond B Biol Sci 2018;373:20170268.

22. Duan L, Zhhu G. Psychological interventions for people affected by the COVID-19 epidemic. Lancet Psychiatry 2020;7:300-2. 